\title{
$\angle S$ Research Suare \\ How staph infections trigger cell death in human immune cells
}

Ruiyuan Yao

Yuhao Chen

Huifang Hao

Zhixin Guo

Xiaoou Cheng

Yuze Ma

Qiang Ji

Xiaoru Yang

Yanfeng Wang

Xihe Li

Zhigang Wang

\section{Video Byte}

Keywords: Cell Communication and Signaling, pyroptosis, Staphylococcus aureus, mTORC1, STAT3. macrophage, mechanism, signaling pathway, Stattic, rapamycin, IL-18, IL-1ß, GSDMD-NT pore structures, Caspase1, NLRP3

Posted Date: February 25th, 2021

DOI: https://doi.org/10.21203/rs.3.rs-276034/v1

License: (a) (1) This work is licensed under a Creative Commons Attribution 4.0 International License.

Read Full License 


\section{Abstract}

Pyroptosis is a self-destruction sequence initiated by cells when infected by pathogens such as bacteria. This programmed cell death helps clear the body of infection, but the molecular mechanism controlling that process has remained a mystery. Now, new research points to the molecules mTORC1 and STAT3 as important participants in pyroptosis in human immune cells. Researchers examined how pyroptosis is initiated in macrophages by S. aureus, the bacteria that cause "staph" infections. Experiments showed that using rapamycin to inhibit $\mathrm{mTORC1}$, a protein that regulates conventional forms of cell death, led to pyroptosis in macrophages. causing the cells to swell with characteristically large bubbles. Further experiments revealed that mTORC1 works in conjunction with STAT3, another cell death protein. Blocking STAT3, using the inhibitor Stattic, also led to pyroptosis. The findings indicate that the mTORC1/STAT3 axis is critical to pyroptosis in human macrophages. Understanding this process could help researchers figure out how pathogens switch pyroptosis off to linger longer in the body. 